\title{
Primary Renal Myxofibrosarcoma in a Woman: A Case Report and Literature Review
}

Sung Han Kim ${ }^{1}$, Yoon Seok Suh ${ }^{1}$, Jung Kwon Kim ${ }^{1}$, Weon Seo Park ${ }^{2}$, Seog Yun Park ${ }^{2}$, Ji Hyeon Lee ${ }^{2}$, Sun Ho Kim ${ }^{3}$, Jae Young Joung ${ }^{1}$, Ho Kyung Seo ${ }^{1}$, Kang Hyun Lee ${ }^{1}$ and Jinsoo Chung ${ }^{1^{*}}$

${ }^{1}$ Department of Urology, Center for Prostate Cancer, National Cancer Center, Goyang Republic of Korea ${ }^{2}$ Department of Pathology, Center for Prostate Cancer, National Cancer Center, Goyang, Republic of Korea ${ }^{3}$ Department of Radiology, Center for Prostate Cancer, National Cancer Center, Goyang, Republic of Korea

*Corresponding author: Chung J, M.D, Ph.D. Center for Prostate Cancer, National Cancer Center, 323 Ilsan-ro, Ilsandong-gu, Goyang-si, Gyeonggi-do, 410-480, Republic of Korea, Tel: +82-31-920-1676; Fax: +82-31-920-1790; E-mail: cjs5225@ncc.re.kr

Rec date: June 06, 2017, Acc date: Oct 10, 2017, Pub date: Oct 22, 2017

Copyright: $\odot 2017 \mathrm{Kim} \mathrm{SH}$, et al. This is an open-access article distributed under the terms of the Creative Commons Attribution License, which permits unrestricted use, distribution, and reproduction in any medium, provided the original author and source are credited.

\begin{abstract}
A 45-year-old previously healthy woman presented to our hospital with a right abdominal mass. Abdominal computed tomography (CT), magnetic resonance imaging, and bone scanning revealed a $15 \mathrm{~cm} \times 10 \mathrm{~cm} \times 14 \mathrm{~cm}$, well-delineated, retroperitoneal tumor originating from the right kidney. No metastases detected. The patient underwent successful extirpation of the renal tumor. The tumor showed $50 \%$ necrosis without perirenal fat infiltration, and it was diagnosed as a high-grade (grade 3) myxofibrosarcoma without nodal positivity, according to the French Federation of Cancer Centers Sarcoma (FNCLCC) grading system. Tumor immunohistochemical staining revealed positive staining for CD34, Ki67, smooth muscle actin (SMA), and cluster of differentiation 68 (CD68), and negative staining for $\mathrm{S} 100$ protein, desmin, mast/stem cell growth factor receptor (c-kit), and melanoma marker antibody (HMB45). Three months post-surgery, follow-up CT revealed no new abdominal metastasis, and the patient is currently receiving routine follow-up without any additional systemic therapy. Myxofibrosarcoma of the kidney is an uncommon soft tissue tumor, and radical surgery is the treatment of choice. Long-term follow-up is recommended because of the tumor's aggressive invasiveness and potential for distant metastasis.
\end{abstract}

Keywords: Kidney neoplasms; Retroperitoneal neoplasms; Ki-67 Antigen; Desmin

\section{Introduction}

Primary renal sarcomas in adults are rare, accounting for about $1 \%$ of all primary renal malignancies [1-3]. While leiomyosarcoma is the most common renal mesenchymal malignancy [2,3], osteosarcoma, angiosarcoma, rhabdomyosarcoma, synovial sarcoma, pleomorphic undifferentiated sarcoma, and fibrosarcoma are also prevalent. Retroperitoneal myxofibrosarcoma (MFS), especially of renal origin, is a more uncommon soft tissue tumor of the elderly [4], seen in less than $3 \%$ of all renal neoplasms [5]. It was previously known as the myxoid variant of malignant fibrous histiocytoma [6], and is regarded as a malignant fibroblastic lesion with variable myxoid stroma. Histologically, MFS can be classified into three grades according to the grading system of the French Federation of Cancer Centers Sarcoma group (FNCLCC) [7]. Intermediate grade (grade 2) and high-grade (grade 3) neoplasms have a local recurrence rate of over $50 \%$, and display high metastatic potential [5]. Primary MFS of the retroperitoneum is very rare, since this type of tumor is usually detected in the extremities of elderly patients. We report here a 45year-old woman with localized MFS who underwent radical nephrectomy and tumor excision. The patient presented no evidence of metastasis or local recurrence 3 months post-surgery. We also discuss the current case in the context of relevant literature to aid in differential diagnosis in future cases.

\section{Case Description}

A 45-year-old previously healthy woman presented with a right abdominal mass at our institution. Abdominal computed tomography (CT) showed a $15 \mathrm{~cm} \times 10 \mathrm{~cm} \times 14 \mathrm{~cm}$, well delineated, retroperitoneal tumor, originating from the right kidney. Kidney MRI further confirmed the abdominal CT findings, and was suggestive of renal cell carcinoma.

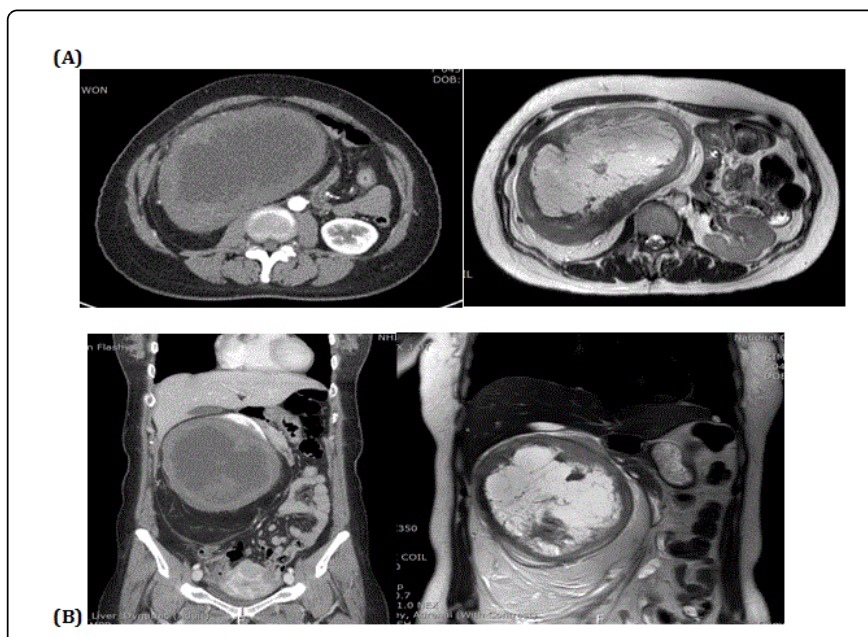

Figure 1: Enhancing computed tomographic and magnetic resonance images of the tumor, (A) axial view (B) coronal view. 
While no evidence of metastasis was found, abdominal CT revealed multiple uterine myomas $(1.5 \mathrm{~cm}$ and $2.8 \mathrm{~cm}$ in size) and several small liver cysts. Chest CT showed no abnormal findings. Further bone scans revealed multiple suspicious abnormal bone uptakes in the 8 th and 11th ribs. Additional radiography revealed degenerative findings (Figure 1). The patient underwent successful extirpation of the tumor and nodal dissection of suspiciously enlarged hilar and pericaval lymph nodes. Tumor invasion into the renal capsule was seen, but without perirenal fat infiltration. The mass was relatively well demarcated, but adhered to the kidney (Figure 2).

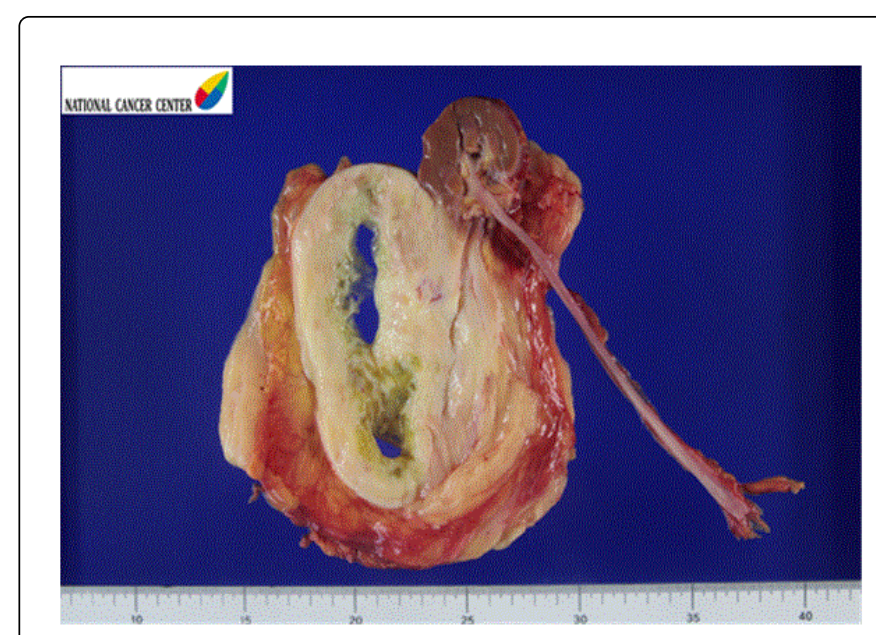

Figure 2: Gross pathology of the tumor section. Tumor section depicting a grayish white solid mass with about $50 \%$ necrosis. The mass was relatively well demarcated, but adhered to the kidney.

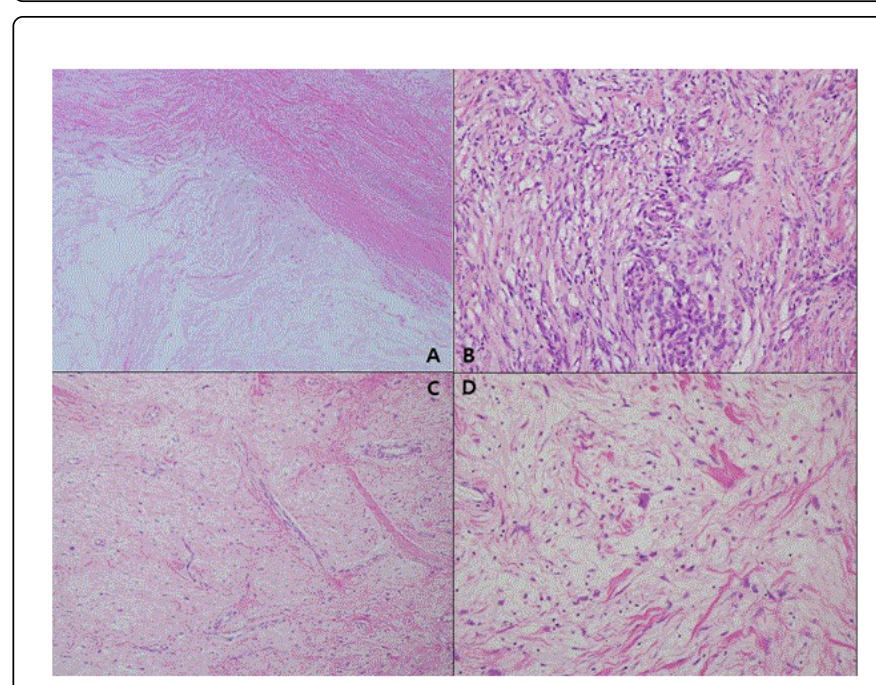

Figure 3: Tumor histopathology. Histopathological tumor images showing (A) nodular growth, with prominent myxoid matrix $(\times 40)$, (B) solid sheets and cellular fascicles of spindled, pleomorphic cells ( $\times 200)$, (C) elongated, curvilinear vasculature $(\times 100),(D)$ frequent multinucleated giant cells with abundant eosinophilic cytoplasm $(\times 200)$.
On sectioning, it appeared as a grayish white solid mass, with 50\% necrosis nodular growth, and a prominent myxoid matrix (Figure $3 \mathrm{~A}$ ). Solid sheets and cellular fascicles of spindled and pleomorphic tumor cells were visible on histopathological examination (Figure 3B). The average mitotic count was seven per 50 high-powered fields, with no atypical mitotic figures. Immunohistochemical staining revealed positivity for CD34, Ki67, SMA, and CD68, and negativity for S100, desmin, c-kit, and HMB45 (Figure 4). A diagnosis of high-grade (grade 3) MFS without nodal positivity and with clear resection margin was made, according to the FNCLCC system. Follow-up CT 3 month's post-surgery revealed no new abdominal or bony metastases. The patient is currently undergoing a routine surveillance protocol for renal cell carcinoma, without any additional systemic therapy.

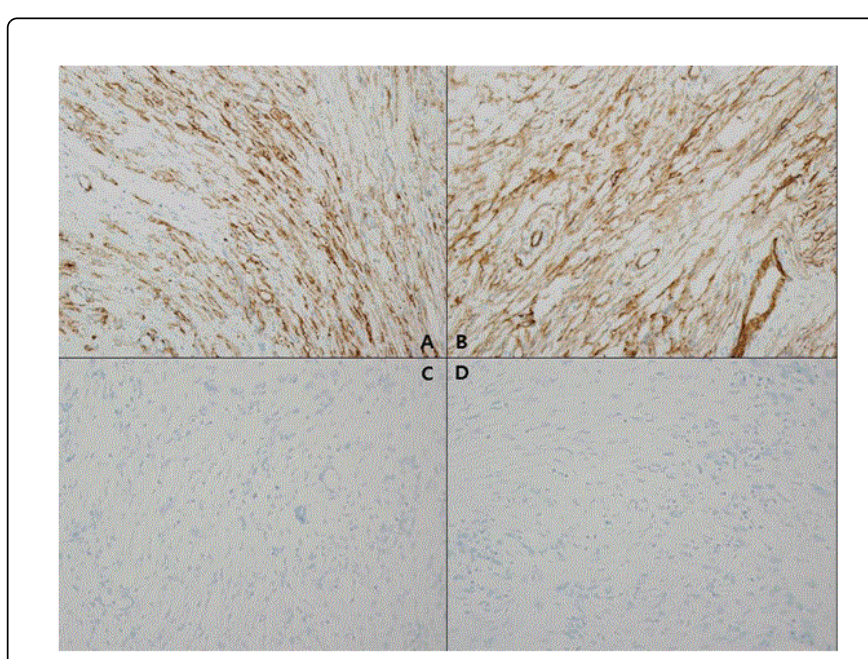

Figure 4: Tumor immunohistochemical images. Tumor sections showing positive staining for (A) smooth muscle actin (SMA), and (B) cluster of differentiation CD34, and negative status for (C) S100, and (D) desmin.

\section{Discussion}

Myxofibrosarcoma is a mesenchymal tumor of fibroblastic origin characterized by a myxoid matrix, and was formerly described as a malignant fibrous histiocytoma. It is usually composed of a variety of spindled, pleomorphic, or sometimes focally epithelioid cells with variable mitotic activity [2]. This tumor constitutes a distinct clinicopathologic entity [5] included in the WHO classification of soft tissue tumors [8]. While MFSs of the visceral organs are uncommon, they have been reported to occur in the heart, the aorta, the thyroid gland, and the brain [2]. Urological MFS tumors of the penis, spermatic cord, kidneys, and prostate are also rare, and less than 30 cases have been reported in the period between 1970 and 2000 [1,5,7-9].

No specific genetic biomarkers exist for MFS, though cytogenetically multiple complex chromosomal aberrations are observed in most cases. A recent study of MFS tumors using next generation sequencing techniques showed intratumoral heterogeneity and mutations in the FGFR3, KIT, KDR, and TP53 genes [3]. Additionally, genetic analysis of cell lines established from MFSs revealed a mutation in the PTEN gene. 
Page 3 of 4

Immunohistochemical biomarkers have been used to characterize disease prognosis in previous studies of MFS. Reactivity for CD34 was observed in $38 \%$ of MFSs [10,11], supporting the fibroblastic nature of the neoplastic cells. Nuclear expression of B-cell lymphoma 2 (bcl2) and protein $\mathrm{p} 53$ serve as proliferative biomarkers, correlating with malignant transformation and aggressive behavior [2,12]. Overexpressed alpha-methylacyl-CoA racemase (AMACR) in MFS can be amplification-driven and is also associated with tumor aggressiveness [13]. Additionally, expression of CD99 has been reported in MFS [14].

For MFS, the most important differential diagnostic entities to be ruled out by immunohistochemistry include sarcomatoid squamous cell carcinoma (positive for cytokeratin, DK5/6, and p63), and melanoma (positive for protein S100/SOX10) [10].

In the present case of localized MFS, immunohistochemical analysis revealed expression of CD34, Ki67, SMA, and CD68 biomarkers, while S100, desmin, c-kit, and HMB45 biomarkers were absent. Gross morphologic findings revealed a vaguely nodular growth pattern, and the presence of an abundant myxoid matrix. A prominent vascular pattern of elongated curvilinear small capillaries, fusiform, round or stellate tumor cells with indistinct cell margins and a striking inflammatory infiltrate especially around vessels and adjacent tumor cells was seen (Figure 3C). Pseudolipoblasts (cells with one large or multiple small cytoplasmic mucin-containing vacuoles) and giant cells with hyperchromatic atypical nuclei and acidophilic cytoplasm were prominent (Figure 3D). Tumor differentiation, mitotic activity, and focal areas of necrosis qualified our lesion as FNCLCC grade 3 MFS.

Prognostic stratification of malignant mesenchymal tumors is based on tumor size, surgical resection margins, and grade $[6,11,15]$. A local recurrence rate of $31 \%$ and a metastasis rate of $17 \%$ have been reported during the 3.5 years of follow-up post-surgery $[16,17]$. Lungs, bones, and lymph nodes are the most common metastatic sites of MFS $[14,16,18]$. Radical surgical eradication of the tumor with sufficient resection of surrounding margins is the recommended treatment option for MFS. Surgery combined with radiotherapy should be considered for all patients diagnosed with grade 2 or grade 3 MFS [18]. Chemotherapy with doxorubicin and ifosfamide has been used in cases of tumor recurrence. MFSs have a better 5-year overall survival rate of $61 \%$ than other types of sarcoma $[16,18]$. In conclusion, we present a rare case of a retroperitoneal MFS in a middle-aged woman, with histopathological and immunohistochemical findings, to help differential diagnosis in future cases. A long-term follow-up plan is recommended due to the high recurrence rate and potential for metastasis in this tumor type.

\section{Ethics Statement}

All study protocols conformed to the ethical guidelines of the "World Medical Association Declaration of Helsinki-Ethical Principles for Medical Research Involving Human Subjects". Written informed consent was obtained from the patient for publication of this case report and any accompanying images. A copy of the written consent is available for review by the journal editor. This case report has been granted an exemption of IRB approval from the ethics committee of the IRB in the National Cancer Center.

\section{Financial Disclosure}

This research did not receive any specific grants from funding agencies in the public, commercial, or not-for-profit sectors.

\section{Author Contributions}

Park WS, Park SY, Lee JH carried out the histopathology and Immunohistochemical analysis. "Sung Han Kim" and "Yoon Seok Suh" are equally contributed for the manuscript as first authors. Chung JS, Park WS, Kim SH, Suh YS conceived of the study, participated in its design and coordination, and helped draft the manuscript. Chung JS and Kim SH carried out the surgery. Suh YS and Kim JK collected all the data. All authors have read and approved the final manuscript.

\section{References}

1. Grignon DJ, Ayala AG, Ro JY, el-Naggar A, Papadopoulos NJ (1990) Primary sarcomas of the kidney - A clinicopathologic and DNA flow cytometric study of 17 cases. Cancer 65: 1611-1618.

2. Val-Bernal JF, Garcia-Gonzalez MR, Mayorga M, Marrero RH, JorgePerez N (2015) Primary renal myxofibrosarcoma. Pathol Res Pract 211: 619-624.

3. Lohberger B, Stuendl N, Leithner A, Rinner B, Sauer S, et al. (2017) Establishment of a novel cellular model for myxofibrosarcoma heterogeneity. Sci Rep 7: 44700.

4. Angervall L, Kindblom LG, Merck C (1977 Myxofibrosarcoma. A study of 30 cases. Acta Pathol Microbiol Scand A) 85A: 127-140.

5. Mentzel T, Calonje E, Wadden C, Camplejohn RS, Beham A, et al. (1996) Myxofibrosarcoma. Clinicopathologic analysis of 75 cases with emphasis on the low-grade variant. Am J Surg Pathol 20: 391-405

6. Weiss SW, Enzinger FM (1977) Myxoid variant of malignant fibrous histiocytoma. Cancer 39(4): 1672-1685.

7. Neuville A, Chibon F, Coindre JM (2014) Grading of soft tissue sarcomas: from histological to molecular assessment. Pathology 46: 113-120.

8. Doyle LA (2014) Sarcoma classification: an update based on the 2013 World Health Organization Classification of Tumors of Soft Tissue and Bone. Cancer 120: 1763-1774.

9. Tearada H, Nagata M, Mugiya S, Ozono S (2012) High-grade myxofibrosarcoma presenting at the spermatic cord after radiotherapy for prostate cancer. BMJ Case Rep.

10. Jo VY, Fletcher CD (2014) WHO classification of soft tissue tumours: an update based on the 2013 (4thedn), Pathology 46: 95-104.

11. Wada T, Hasegawa T, Nagoya S, Kawaguchi S, Kaya M, et al. (2000) Myxofibrosarcoma with an infiltrative growth pattern: a case report. Jpn J Clin Oncol 30(10): 458-462.

12. Drachenberg CB, Ioffe OB, Castellani RJ, Papadimitriou JC (1997) Nuclear bcl-2 staining in tumors. Am J Clin Pathol 108: 479.

13. Li CF, Fang FM, Lan J, Wang JW, Kung HJ, et al. (2014) AMACR amplification in myxofibrosarcomas: a mechanism of overexpression that promotes cell proliferation with therapeutic relevance. Clin Cancer Res 20: 6141-6152.

14. Illueca C, Machado I, Cruz J, Almenar S, Noguera R, et al. (2012) Pleomorphic hyalinizing angiectatic tumor: a report of 3 new cases, 1 with sarcomatous myxofibrosarcoma component and another with unreported soft tissue palpebral location. Appl Immunohistochem Mol Morphol 20: 96-101.

15. Huang HY, Lal P, Qin J, Brennan MF, Antonescu CR (2004) Low-grade myxofibrosarcoma: a clinicopathologic analysis of 49 cases treated at a single institution with simultaneous assessment of the efficacy of 3-tier and 4-tier grading systems. Hum Pathol 35: 612-621.

16. Sanfilippo R, Miceli R, Grosso F, Fiore M, Puma E, et al. (2011) Myxofibrosarcoma: prognostic factors and survival in a series of patients treated at a single institution. Ann Surg Oncol 18: 720-725.

17. Haglund KE, Raut CP, Nascimento AF, Wang Q, George S, et al. (2012) Recurrence patterns and survival for patients with intermediate- and high-grade myxofibrosarcoma. Int J Radiat Oncol Biol Phys 82: 361-367.

18. Hong LNJ, Hornicek FJ, Raskin KA, Yoon SS, Szymonifka J, et al. (2013) Prognostic Factors and Outcomes of Patients with Myxofibrosarcoma Ann Surg Oncol 20: 80-86. 